DeepConnection: Classifying Relationship State from Images of Romantic Couples

\author{
Maximiliane Uhlich ${ }^{1}$, Daniel Bojar ${ }^{2}$ \\ ${ }^{1}$ Zurich, Switzerland, ${ }^{2}$ Basel, Switzerland
}

Corresponding Author's contact information:

Daniel Bojar, $\mathrm{PhD}$

E-mail: daniel@bojar.net 


\title{
CLASSIFYING RELATIONSHIP STATE FROM IMAGES OF COUPLES
}

\begin{abstract}
Detecting relationship state and quality in romantic couples is an important endeavor for relationship research, couple therapy, and of course couples themselves. Yet current methods to achieve this are intrusive, asynchronous, plagued by ceiling effects, and only assess subjective responses to questionnaires while trying to capture the objective state of a relationship. According to social appraisal theory, human beings rely on emotional responses to assess interpersonal situations, a key element for relationship functioning in couples. Using couples is particularly advantageous as strong emotional reactions are triggered in romantic relationships. Prior research has repeatedly demonstrated that emotion expression in couple interactions is related to relationship satisfaction. Here, we employ deep learning methods to assess the relationship state of romantic couples from images via facial and bodily emotion expression and other features. Our model, DeepConnection, comprises pretrained residual neural networks, spatial pyramid pooling layers, and power mean transformations to extract relevant features from images for binary classification. With this, we achieved an average accuracy of nearly $97 \%$ on a separate validation dataset. We also engaged in model interpretation using Gradient-weighted Class Activation Mapping (Grad-CAM) to identify which features allow DeepConnection to detect relationship state. To demonstrate generalizability and robustness, we used DeepConnection to analyze videos of couples exhibiting a range of different postures and facial expressions. The work presented here could inform couples, advance relationship research, and find application in couple therapy to assist the therapist. (234 words)
\end{abstract}

Keywords: deep learning, relationship research, computer vision, neural network, classification 


\section{CLASSIFYING RELATIONSHIP STATE FROM IMAGES OF COUPLES}

\section{Introduction}

Conscious as well as unconscious emotion expression, gestures, and body posture are highly important cues to any human social interaction, shaping relationship processes (Gable, Reis, Impett, \& Asher, 2004) and coordinating reactions (Keltner \& Haidt, 2001). Individuals tend to express positive affect when feeling happy and negative affect when being upset (Ekman \& Friesen, 1975). Individuals rely on both face and body to encode emotions, with facial and bodily expressions tending to be congruent (Gu, Mai, \& Luo, 2013), to understand what a person might experience in a certain moment, and to predict another person's potential behavioral tendencies. Using the body and face for emotion expression, people can signal disapproval during disagreements, triggering a desirable reaction such as affection or reducing undesirable behaviors during social interactions (Keltner \& Haidt, 2001).

People tend to pay more attention to faces when reading emotions (Kret, Pichon, Grèzes, \& de Gelder, 2011) but the perception of facial expressions is influenced by bodily expressions (Gu et al., 2013). There are several facial cues individuals use for information, stemming from eyes, eyebrows, mouth, cheeks, and nose (Ekman \& Friesen, 1975). Body posture characterizes emotions through closeness and distance toward another person. Being angry is usually accompanied by dominance behavior and approach tendencies for instance. Conversely, feeling fearful is associated with more submissive behavior and avoidance tendencies (Kurien, 2010). Gestures can be made voluntarily or involuntarily and, for instance, involve arms, fingers, head, and legs. Hands are often used to underline statements during a conversation (Ekman \& Friesen, 1975). Being relaxed with arms akimbo or leaned forward signals attention and openness.

Conversely, clenched hands suggest stress and the wringing of hands often implies nervousness (Kurien, 2010). 


\section{CLASSIFYING RELATIONSHIP STATE FROM IMAGES OF COUPLES}

Emotions usually arise during intense social interactions (Butler, 2011). One of the most important social functions of experiencing, displaying, and observing emotions is to structure each other's behavior and help select an appropriate response which can be similar or complementary to the counterpart's behavior (Keltner \& Haidt, 2001). According to social appraisal theory, emotional responses in an interaction between partners guide the evaluation of the meaning of the situation (Parkinson, 2001).

Within the context of romantic relationships, emotional content and accompanying vulnerabilities are much more prevalent than with individuals that are less close (Barrett, Robin, Pietromonaco, \& Eyssell, 1998). Furthermore, displaying emotions toward the partner is much more socially accepted than in a professional context, where masking one's emotions is considered appropriate (Grandey, Rafaeli, Ravid, Wirtz, \& Steiner, 2010). In romantic relationships, the impact of the partner's behavior is thus particularly powerful regarding emotional responses (Johnson, Hunsley, Greenberg, \& Schindler, 2006). As attachment theory (Ainsworth \& Bell, 1970; Bowlby, 1988) suggests, the partner becomes the primary attachment figure in adulthood (Shaver, Hazan, \& Bradshaw, 1988), explaining the strong emotional reactions couples can elicit from each other, as feeling rejected by the partner is very threatening (Johnson et al., 2006).

Communication, verbal and non-verbal, is one of the strongest predictors for relationship satisfaction. For instance, frequently expressing contempt by eye rolling during conflicts predicts divorce within the next six years with an accuracy of $83 \%$ (Gottman, Coan, Carrere, \& Swanson, 1998). Negative emotions are usually triggered when a situation is perceived as a threat to the self or the relationship (Fischer \& Manstead, 2016), which is usually the case during conflicts (Carrere \& Gottman, 1999). Frequent and destructive conflicts undermine relationship 


\section{CLASSIFYING RELATIONSHIP STATE FROM IMAGES OF COUPLES}

satisfaction (Gottman et al., 1998), with couples that remain in negative affective states for extended periods having poorer relationship outcomes (Gottman, Murray, Swanson, Tyson, \& Swanson, 2002). Expressing anger toward the partner tends to go along with social distancing (Roseman, Wiest, \& Swartz, 1994), while reacting adaptively during a conflict makes the partner more likely to respond in a reciprocal manner (Keltner \& Haidt, 2001). Positive emotions, such as desire, compassion or love, facilitate the formation or maintenance of close bonds (Shiota, Campos, Keltner, \& Hertenstein, 2004). Thus, disclosing emotions serves a key function in romantic relationships, enabling constructive interpersonal engagement as well as predicting intimacy and relationship satisfaction (Laurenceau, Barrett, \& Rovine, 2005).

The current gold standard for measuring affect during couple interactions on video is The Specific Affect Coding System (SPAFF; Coan \& Gottman, 2007), which proved to be very useful in predicting relationship outcomes and was used in dozens of studies (e.g. Carstensen, Gottman, \& Levenson, 1995; Cohan \& Bradbury, 1997; Gottman et al., 2003). However, the powerful predictions notwithstanding, the SPAFF is time-consuming. Coders need to train for several weeks to learn the Facial Action Coding System (FACS; Ekman \& Friesen, 1978) and practice coding with videos to eventually arrive at a high interrater reliability. Afterwards, videos of interest will be usually coded in one second intervals, which can take time, depending on video length and sample size. Observational methods are considered the most objective assessment of human behavior, with lower social desirability than self-report measures and less bias, as humans are not particularly accurate in predicting their own behaviors, especially when these are unconscious (Floyd, Baucom, Godfrey, \& Palmer, 1998). Based on the SPAFF research, emotion expression during couple interactions is an important predictor for relationship outcomes. Yet, as mentioned above, there is an abundance of visual information such as gestures 


\section{CLASSIFYING RELATIONSHIP STATE FROM IMAGES OF COUPLES}

or body posture, in addition to facial information, to encode emotions arising in interacting couples, which are not yet fully utilized for relationship research.

Computer vision, the analysis of image and video data, has recently transitioned from a hand-picked, optimized feature crafting workflow to deep learning (Voulodimos, Doulamis, Doulamis, \& Protopapadakis, 2018). Automatically engineering millions of predictive features from images with neural networks, deep learning techniques rapidly surpassed previous state-ofthe-art results in computer vision. The main building block used for computer vision models today is the convolutional neural network ( $\mathrm{CNN}$, consisting of convolutional blocks or layers) (Lecun, Bottou, Bengio, \& Haffner, 1998). Iteratively applying different activation filters to image pixel intensities, CNNs successively increase the image area being considered and can thereby detect features such as edges or even eyeballs etc. Other, more elaborate, neural network designs capitalizing on and extending CNNs such as residual neural networks (ResNets) (He, Zhang, Ren, \& Sun, 2015), densely connected neural networks (DenseNets) (Huang, Liu, van der Maaten, \& Weinberger, 2016) or U-Nets (Ronneberger, Fischer, \& Brox, 2015) have been developed in recent years. Model architectures such as these find wide application, for instance in the measurement of image depth (Z. Li et al., 2019) or for semantic segmentation, relevant for autonomous vehicles (Xu, Li, Xie, Ren, \& Xie, 2019).

Another area in which CNN-based deep learning methods have been successfully applied is emotion recognition (Dachapally, 2017; Mohammadpour, Khaliliardali, Hashemi, \& AlyanNezhadi, 2017), closely related to the aim of this work. Since emotions can be reliably detected in facial expressions (Li \& Deng, 2018), our aim was to classify the relationship state of romantic couples from images using a CNN-based deep learning model. This approach relies upon the visible demonstration of affection or dissatisfaction between romantic partners via 


\section{CLASSIFYING RELATIONSHIP STATE FROM IMAGES OF COUPLES}

emotions recognizable in facial expressions or other body expressions, consciously or subconsciously. Potentially, this could circumvent biases such as ceiling effects (Funk \& Rogge, 2007) in the self-reporting of relationship satisfaction, offer a vastly more efficient data collection regime than survey-based methods (especially in the case of automated video analysis), and an unobtrusive method, as the couple is not primed by questions regarding their relationship satisfaction (Webb, Campbell, Schwartz, \& Sechrest, 1966).

After web scraping relevant images of couples labeled as happy or unhappy, we therefore built the model DeepConnection to classify the relationship state of romantic couples from images. As transfer learning, in which an already trained base model is used to initialize another model, has been shown to perform excellently in computer vision by transferring learned features (Yosinski, Clune, Bengio, \& Lipson, 2014), we made use of a pretrained ResNet-type model as our base model. As this base model has been already trained on over one million images from the ImageNet competition (Deng et al., 2009), we can use considerably fewer images to fine-tune the model to our purpose. We further optimized this base model with additional modules to construct the superior DeepConnection model. Then, we analyzed the classification mechanism of DeepConnection using gradient-based techniques to visualize model saliency heatmaps. Assessing the performance of DeepConnection on video frames of couples absent from the training data further demonstrated the robustness and relevance of our constructed relationship state classifier as well as its potential for deployment in real-world scenarios. 


\section{CLASSIFYING RELATIONSHIP STATE FROM IMAGES OF COUPLES}

\section{Method}

\section{Data Collection}

Images featuring couples were gathered by web scraping, using the Google Images Download Python script (https://github.com/hardikvasa/google-images-download). For each search term, a maximum of 100 images were downloaded. For happy couples, the search terms ' $\{$ happy, laughing, loving, healthy, romantic, dedicated, committed, relaxed, content, satisfied $\}$ couple’ were used, while '\{unhappy, sad, angry, disappointed, fighting, frustrated, annoyed, mad, dysfunctional, estranged, dissatisfied \} couple' were used to collect images of unhappy couples. Subsequently, all images were manually inspected and images which displayed only small parts of the bodies of couples, cartoons, single persons, artificial or ambiguous expressions as well as extremely low resolution images were discarded. Training data consisted of 640 images of couples labeled as happy and 435 images labeled as unhappy couples. Validation data consisted of 147 images of happy and 95 images of unhappy couples, randomly selected from the full dataset.

\section{Data Preprocessing}

All images were resized to a size of 512 by 512 pixels. Images in the training set were augmented with the ImgAug Python library (https://github.com/aleju/imgaug) by a random order of partial horizontal flipping (50\%), cropping, partial application of Gaussian blur (50\%), application of additive Gaussian noise, changes in intensity, hue, saturation and contrast as well as rotation, shearing, translation, and scaling. Afterwards, images were converted to tensors and normalized with ImageNet statistics (Deng et al., 2009). 


\section{CLASSIFYING RELATIONSHIP STATE FROM IMAGES OF COUPLES}

\section{Model Training}

Models were trained in PyTorch (Paszke et al., 2017) with mixed precision training using the Apex library (https://github.com/nvidia/apex). All models used a batch size of 48, a starting learning rate of 0.0001 and an early stopping criterion of five consecutive epochs without decrease in validation loss (Prechelt, 2012). Adam was used as an optimizer (Kingma \& Ba, 2014) with a weight decay value of 0.1 , cross-entropy loss as a loss function and a cosine learning rate annealing schedule over 15 epochs to decrease the learning rate over time (Loshchilov \& Hutter, 2016). As a model base, the convolutional part of a pretrained ResNet-34 model was used (He et al., 2015). For the SPP model and DeepConnection, the last adaptive average pooling layer of this model was exchanged for a spatial pyramid pooling layer (He, Zhang, Ren, \& Sun, 2014) with spatial bins of size [8x8], [4x4], [2x2] and [1x1], resulting in 43520 features. All features were concatenated and, for DeepConnection, power mean transformation (Zhang \& Wu, 2019) was added prior to the first fully connected layer after the spatial pyramid pooling layer, doubling the number of features. These features were then passed on to a number of fully connected layers with ReLU (rectified linear unit) activations (Nair \& Hinton, 2010), regularized by dropout (0.5) and batch normalization layers (Ioffe \& Szegedy, 2015; Srivastava, Hinton, Krizhevsky, Sutskever, \& Salakhutdinov, 2014). Linear layers were initialized by using Xavier initialization (Glorot \& Bengio, 2010). Models were trained by backpropagating gradients in the newly added part of the model until early stopping. All models were trained on a single NVIDIA ${ }^{\circledR}$ Tesla $^{\circledR}$ K80 GPU.

\section{Analysis of Videos of Romantic Couples}

In total, 20 videos were gathered from an open source platform

(https://www.shutterstock.com/de/video) with equal proportions of videos containing happy and 


\section{CLASSIFYING RELATIONSHIP STATE FROM IMAGES OF COUPLES}

unhappy couples. From each video, 12 representative video frames were manually chosen. After applying the same preprocessing steps as for validation images, frames of a given video were classified by all trained models. Accuracy was averaged across all frames of one video to determine the couple-specific accuracy and averaged across all couples to determine the classspecific and overall accuracy on video frames.

\section{Results}

\section{Building a Deep Learning Model to Detect the Relationship State of Romantic Couples}

Images of couples contain a cornucopia of information, ranging from body posture (both by themselves and relative to the partner) and gestures to facial expressions. Since most of these characteristics are subconscious forms of behavior, images of couples seem perfectly poised to potentially yield an honest assessment of their relationship state devoid of societal bias, if one has access to several representative images. Here, we used the highly successful residual neural network (ResNet) architecture (He et al., 2015) as the model base to predict whether an image depicted a happy or unhappy couple. ResNets consist of residual blocks comprising convolutional layers (constructing and detecting ever-larger features in images) and skipconnections, which allow the network to skip a whole residual block if it does not improve the result. After initial experimentation, we chose a pretrained ResNet model with 34 layers (ResNet-34) as the basis for our binary classifier, combined with a block of fully connected layers which yielded the probabilities for each class and which were trained with the gathered image data. 


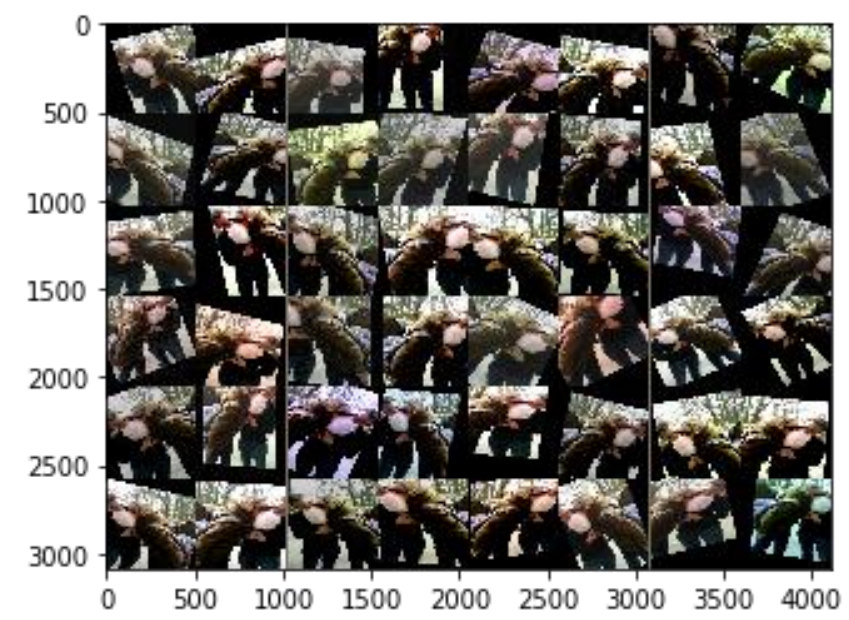

Figure 1. Exemplary batch of training images seen by models after data augmentation. After resizing images to $512 \times 512$ pixels, images were subjected to a wide array of data augmentation techniques. These comprised a random order of horizontal flipping, cropping, application of Gaussian blur and additive Gaussian noise, changes in intensity, hue, saturation and contrast as well as rotation, shearing, translation, and scaling. Subsequently, images were normalized with ImageNet statistics, shuffled and stored together with their class labels in groups of 48. Here, only one representative image is shown which underwent 48 different data augmentation routes representative for our training data.

Especially with limited amounts of available training data, data augmentation is crucial. Applying specific distortions such as rotation, cropping or the addition of noise to images can increase the accuracy of a model by providing it with augmented training data and improve the generalizability as well as robustness of a model (Mikolajczyk \& Grochowski, 2018). We used a wide array of augmentation techniques (see Methods, Fig. 1), some of which were only applied to a random fraction of the training data. Thereby, we intended to avoid narrowly focusing on 


\section{CLASSIFYING RELATIONSHIP STATE FROM IMAGES OF COUPLES}

image peculiarities present in our dataset but which could be absent from real-world contexts and thus achieved high validation accuracy (Table 1).

Table 1

Performance metrics of deep learning models used to classify relationship state of romantic couples

\begin{tabular}{ccccccc}
\hline Model Base & \multicolumn{2}{c}{ ResNet-34 } & \multicolumn{2}{c}{ SPP } & \multicolumn{2}{c}{$\begin{array}{c}\text { SPP-PMT } \\
\text { (DeepConnection) }\end{array}$} \\
& $M$ & $S D$ & $M$ & $S D$ & $M$ & $S D$ \\
\hline Cross-Entropy Loss & 0.2358 & 0.0254 & 0.1539 & 0.0153 & $\mathbf{0 . 1 3 0 8}$ & 0.0134 \\
Accuracy & 0.9170 & 0.0072 & 0.9535 & 0.0099 & $\mathbf{0 . 9 6 7 7}$ & 0.0035 \\
Precision & 0.9373 & 0.0303 & 0.9632 & 0.0344 & $\mathbf{0 . 9 8 0 8}$ & 0.0114 \\
Recall & 0.8626 & 0.0461 & 0.8816 & 0.1042 & $\mathbf{0 . 9 6 2 5}$ & 0.0142 \\
\hline
\end{tabular}

Note. Each model base was connected to a classifier head consisting of fully connected layers. Cross-entropy loss, accuracy, precision, and recall were assessed on the validation dataset across five training runs for each model. Numbers in bold indicate the superior metric value for each presented metric. Values are presented as mean $(M)$ and standard deviation $(S D)$.

Given that ResNet-34 was originally trained on images of size 224x224 pixels and many essential details might be lost at this resolution, we exchanged the final adaptive average pooling layer with a spatial pyramid pooling (SPP) layer (He et al., 2014). This layer divides the output of the last convolutional layer into a given number of spatial bins and conducts a max-pooling operation on all spatial bins for all convolutional channels and concatenates the result as new 


\section{CLASSIFYING RELATIONSHIP STATE FROM IMAGES OF COUPLES}

features. Next to yielding a fixed size representation regardless of image size and thereby allowing flexible usage, SPP layers are also robust to object deformations. With this, we could use higher-resolution images of size $512 \times 512$ pixels with different numbers of spatial bins. A combination of bins of size [8x8], [4x4], [2x2], and [1x1] resulted in best performance, substantially improving validation loss and accuracy (Table 1). We also tried to extend SPP by concatenating the average-pooling of spatial bins, in addition to the max-pooling to capture systematic effects, yet saw no further increase in model performance.

Nonlinearity in our model mainly stemmed from activation and pooling layers. To increase and diversify nonlinearity, we introduced power mean transformation (PMT: Zhang \& $\mathrm{Wu}, 2019$ ) into our model between the SPP layer and the subsequent fully connected layer and observed an increase in validation accuracy. In contrast to Zhang \& Wu, we found no additional performance increase in adding another PMT prior to the first convolutional layer. We also confirmed that concatenating the original SPP features in addition to the power meantransformed SPP features did not further improve model performance. These findings resulted in our final model, DeepConnection (Fig. 2).

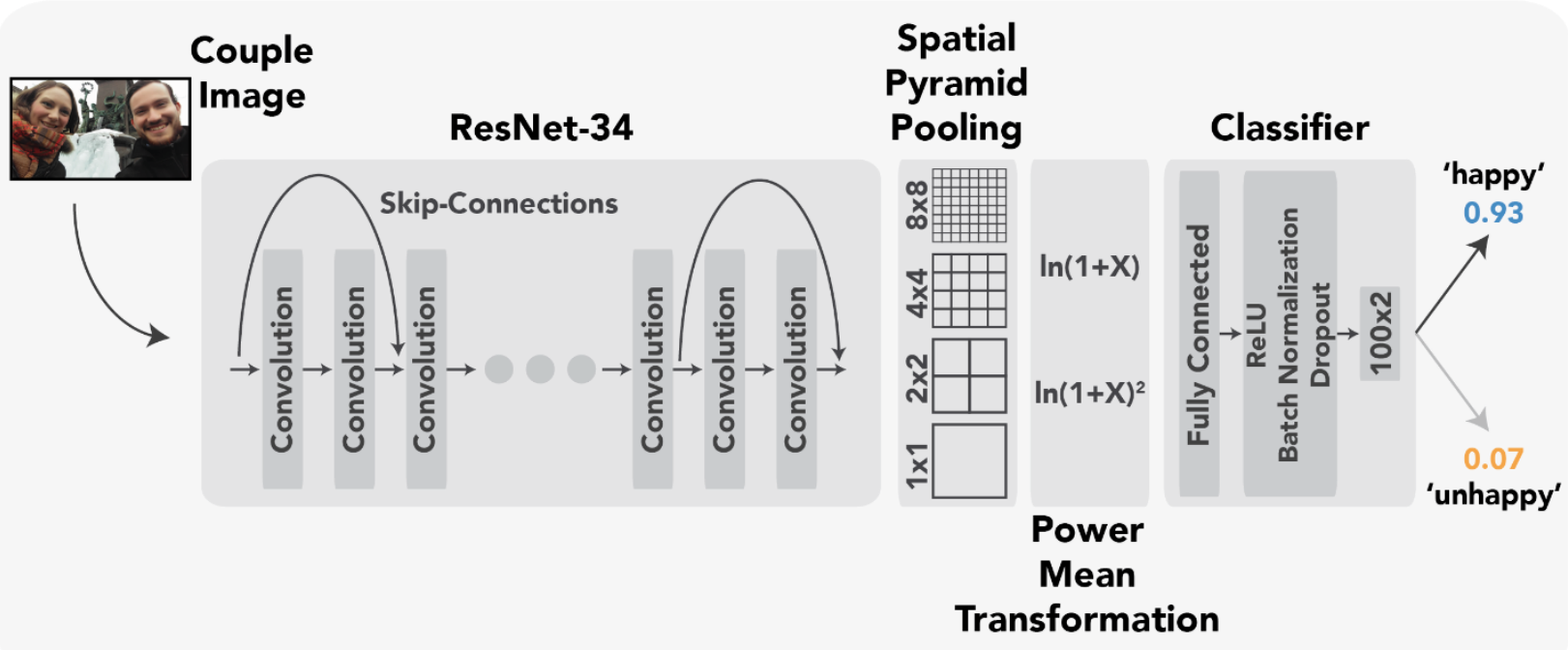




\section{CLASSIFYING RELATIONSHIP STATE FROM IMAGES OF COUPLES}

Figure 2. Model architecture of DeepConnection. After resizing, data augmentation and normalization, images were used as inputs for the convolutional part of a pretrained ResNet-34 model. Subsequent to the last convolutional layer, a spatial pyramid pooling layer extracts a fixed-size representation from the convolutional output. For this, the convolutional channels are divided into [8x8], [4x4], [2x2], [1x1] spatial bins and max-pooled. This concatenated representation is subjected to power mean transformation and passed into a fully connected layer. The output of this layer undergoes a ReLU activation layer, a batch normalization layer, and a dropout layer until it reaches the final fully connected classification layer which yields two values, indicating the respective class probability.

\section{Analyzing the Representation of Relationship States in DeepConnection}

Despite their performance, deep learning models are frequently disparaged as 'black boxes', in which classification decisions cannot be rationalized. Yet many analysis methods can unravel the inner workings of a deep learning model. One of them is Gradient-weighted Class Activation Mapping (Grad-CAM), which yields a heatmap indicating the saliency of image areas for both classes, respectively (Selvaraju et al., 2016). When applying Grad-CAM to our trained models, we could identify differences in model behavior and salient feature regions between models (Fig. 3). 


\section{CLASSIFYING RELATIONSHIP STATE FROM IMAGES OF COUPLES}

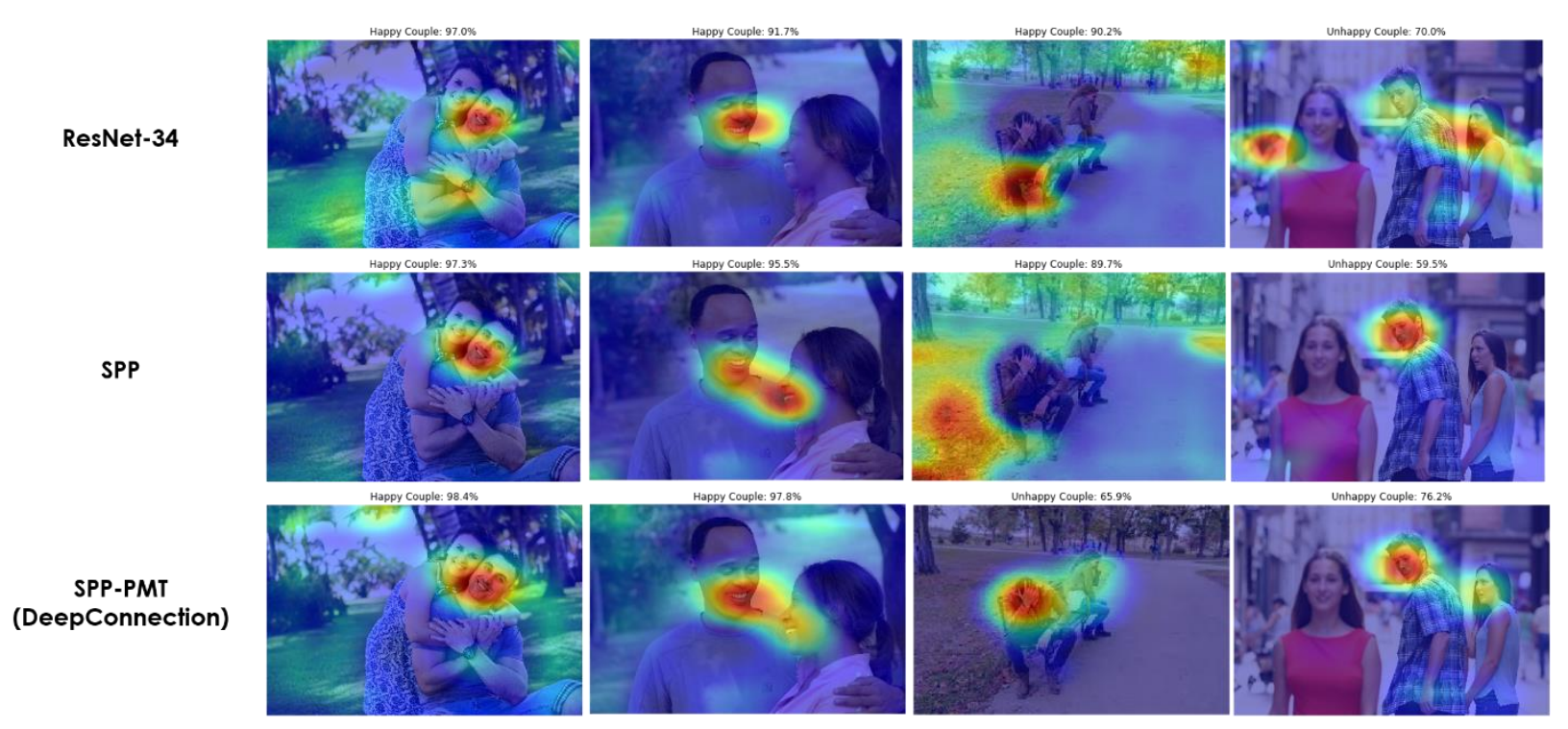

Figure 3. DeepConnection model interpretation using Grad-CAM. Four representative images of couples not used in training (two labeled as happy and two as unhappy) were used as inputs to trained models. All trained models used a classification head consisting of fully connected layers, activation layers, and regularization. For the model bases, a standard ResNet-34 model, a ResNet-34 model with spatial pyramid pooling instead of adaptive averaging pooling (SPP), and a ResNet-34 model with spatial pyramid pooling and subsequent power mean transformation (SPP-PMT, DeepConnection) were used. Gradients at the last convolutional layer were used to generate class-specific saliency heatmaps and layered on top of the original images, together with the predicted class label and the model confidence in percent.

One immediate observation was the importance of faces for the evaluation of relationship state by all models. The lower half of the face, especially the mouth region, seemed to be most relevant for classification. For unhappy couples, this focus seemed to be spread more broadly, encompassing the whole face, which might indicate a higher variability in expression. In general, unhappy couples were harder to classify than happy couples for all models. This was 


\section{CLASSIFYING RELATIONSHIP STATE FROM IMAGES OF COUPLES}

corroborated by a higher precision in the classification of happy couples (Table 1), for which the presence of a smile or grin might be already highly indicative of the class label 'happy couple'. Another interesting observation was that the model confidence in the correct class prediction on average increased in order of increasing model performance as indicated in Table 1.

Concurrently, the correct salient image area seemed to increase from ResNet-34 to

DeepConnection, which might offer an explanation for its superior performance as it evaluated more relevant information.

The robustness of more sophisticated models also seemingly increased. The face of the woman in image two is slightly askew, possibly preventing ResNet-34 to recognize it as a valuable source of information, whereas both the SPP model as well as DeepConnection utilized both faces for classification. The same could be observed in image four, in which only DeepConnection resulted in a clear focus on both faces. Notably, here a third person (who seems to be happy) is seen in the picture and was also independently recognized as happy by the SPP model and DeepConnection (Appendix Fig. 1-3). Despite this confounding factor, all three trained models were able to correctly identify the couple as unhappy, demonstrating their robustness.

An interesting case can be seen in image three, which was mislabeled by ResNet-34 and the SPP model with high model confidence. Here, both faces are obscured, potentially explaining the poor performance of these models. Only DeepConnection was still able to correctly ascertain the unhappy state of this couple by analyzing both partners. This also illustrated that, in absence of clearly visible facial features, DeepConnection utilized information from the upper bodies of the couple, a region important for posture, stance, and emotion expression (Luginbuehl \& Schoebi, 2019). Relying on different salient regions could endow DeepConnection with higher 


\section{CLASSIFYING RELATIONSHIP STATE FROM IMAGES OF COUPLES}

degrees of flexibility and robustness than the other models tested here and could explain its high level of performance.

Additionally, we analyzed the state of activation at the final fully connected layer of DeepConnection, which determined class probabilities, by plotting incoming activations of representative images together with the trained layer weights (Appendix Fig. 4-5). From this, we concluded that a distinct region of the state-of-activation plot was responsible for the classification of happy couples (lower righthand corner) for both the SPP model as well as DeepConnection. Notably, no distinct activation pattern could be found for unhappy couples in the SPP model. Rather, the classification as 'unhappy' seemed to rely predominantly on the depletion of 'happy' characteristics, which might explain the superior model performance with regard to classifying happy couples. Yet DeepConnection exhibited dedicated activation nodes for unhappy couples in the upper lefthand corner (Appendix Fig. 5), potentially inducing an improved model performance.

\section{Testing DeepConnection on Video Data of Couples}

To test the robustness of DeepConnection to image details, and thereby assess its potential for application in real-world contexts, we collected videos of couple interactions and classified video frames. The strength of this approach is that, while the overall video interaction might be enacted, single frames may capture individuals between movements and in less idealized postures than captured in stock images, infusing them with more realism. By averaging classification accuracy across multiple frames, we could assess the performance of DeepConnection for a given couple in a range of different spatial arrangements (Table 2). 


\section{CLASSIFYING RELATIONSHIP STATE FROM IMAGES OF COUPLES}

Table 2

Comparison of model accuracy in the classification of videos featuring happy and unhappy couples

\begin{tabular}{|c|c|c|c|c|c|c|}
\hline \multirow[t]{2}{*}{ Model Base } & \multicolumn{2}{|c|}{ ResNet-34 } & \multicolumn{2}{|c|}{ SPP } & \multicolumn{2}{|c|}{$\begin{array}{c}\text { SPP-PMT } \\
\text { (DeepConnection) }\end{array}$} \\
\hline & $M$ & $S E$ & $M$ & $S E$ & $M$ & $S E$ \\
\hline Accuracy on Happy Couples & 0.5250 & 0.1261 & 0.8166 & 0.0774 & 0.8750 & 0.0485 \\
\hline Accuracy on Unhappy Couples & 0.7084 & 0.1281 & 0.8083 & 0.0714 & 0.8416 & 0.0548 \\
\hline Overall Accuracy & 0.6167 & 0.0900 & 0.8125 & 0.0513 & 0.8583 & 0.0358 \\
\hline
\end{tabular}

Note. Each model base was connected to a classifier head consisting of fully connected layers.

Classification accuracy was averaged for each video (12 frames) and then averaged across videos for the respective class or all videos. In total, 10 videos of happy couples and 10 videos of unhappy couples were tested. Numbers in bold indicate the superior metric value for each presented metric. Values are presented as mean $(M)$ and standard error $(S E)$.

Again, DeepConnection performed better than both ResNet-34 by itself or combined with a spatial pyramid layer. Similar to our validation dataset, the largest impact in terms of model improvement seemed to have come from the SPP layer, with an additional, smaller, boost from the subsequent PMT. The overall accuracy values when classifying these video frames were lower than the validation accuracies reported in Table 1 . This could reflect the increased difficulty to accurately recognize a wide range of e.g. facial expressions for each couple, in contrast to the idealized postures observed in stock images. However, an average accuracy of 


\section{CLASSIFYING RELATIONSHIP STATE FROM IMAGES OF COUPLES}

above $85 \%$ in the case of DeepConnection still would allow for high confidence in model predictions and indicates the potential of DeepConnection to analyze real-time interaction of couples.

\section{Discussion}

Detecting and analyzing the relationship state of romantic couples has been the central focus in relationship research since its conception. As human relationships represent an extremely complex phenomenon, many different methods analyzing various aspects of romantic relationships have to be utilized to fully capture and understand this phenomenon. In addition to copious amounts of existing data stemming from surveys, interviews, and behavioral analysis, we have focused on visual information in the form of images here to capitalize on the rich trove of information present in body language and facial expressions by combining deep learning and relationship research. Our final model DeepConnection extracted enough information from images of romantic couples to classify them into states of happy or unhappy relationships with high accuracy (Table 1). This performance was also transferable to video data of couples never seen by the model before, without manual video coding (Table 2). Additionally, we ensured DeepConnection does not suffer the 'black box' fate most models fall into by engaging in model interpretation using Grad-CAM and state-of-activation plots (Fig. 3, Appendix Fig. 4).

In line with social appraisal theory (Parkinson, 2001), DeepConnection used cues related to emotion expression in interacting couples to derive meaning from the depicted situation and classify if couples are happy or unhappy. Based on the heatmaps (Figure 3), facial emotion expression was one of the main features used for the final prediction, in line with Ekman and

Friesen (1975) identifying the importance of facial expressions when experiencing emotions. For 


\section{CLASSIFYING RELATIONSHIP STATE FROM IMAGES OF COUPLES}

happy couples, DeepConnection focused mostly on the lower face (e.g. their smile), while for unhappy couples attention was spread more broadly. Indeed, happiness is displayed mostly in the lower face, with the brow or forehead usually not involved, in contrast to situations involving negative emotions. Furthermore, there are much more negative emotions (e.g. sadness, fear, anger, contempt, disgust) than positive ones (Ekman \& Friesen, 1978). DeepConnection's focus on the face is similar to human processing of emotions, as we also tend to rely mostly on the face, which can be measured in higher associated amygdala activation in comparison to other bodily expressions (Kret et al., 2011).

Although most of the pictures classified as unhappy depict some negative emotion, negative affect does not always impair a relationship and can even promote intimacy (Kashdan, Volkmann, Breen, \& Han, 2007). Potentially detecting these subtle differences in negative affect could partially explain the high levels of accuracy of DeepConnection. Soft emotions, such as sadness or anxiety, are more likely to elicit caring behavior and support from the partner, in contrast to anger for instance (Fischer \& Manstead, 2016). Expressing emotions appropriate for the context helps in regulating the physical stress reaction (e.g. lower cortisol levels) when a person's goal is rejected and responding with anger can be adaptive (Kirschbaum, Pirke, \& Hellhammer, 1993). Conflicts are part of relationships and their impact on relationship satisfaction tends to depend on conflict frequency, the way the conflict is managed, and if couples are able to reconnect afterwards (Laursen \& Hafen, 2009). Future research should use DeepConnection to investigate longer periods of conflict discussions and analyze how the interaction of the couple evolves, as the ability to quickly regulate emotions, recover from psychological distress, and emotional flexibility (Houben, Van Den Noortgate, \& Kuppens, 2015) are associated with higher well-being and interpersonal functioning (Gross \& John, 2003). 


\section{CLASSIFYING RELATIONSHIP STATE FROM IMAGES OF COUPLES}

Previous work has reached high levels of accuracy in classifying basic emotions from images (Mohammadpour et al., 2017). Yet this differs from the presented work in several important aspects. Images used for training by Mohammadpour and colleagues (2017) were faces of single persons in front of uniform backgrounds. Here, we relied on images of romantic couples and could demonstrate that information from both partners were relevant for our classification model (Fig. 3). The additional presence of single persons, while detected by DeepConnection, could be separated from the identification of relationship state (Appendix Fig.

3). This goal of detecting relationship state is also distinctly different from classifying basic emotions. Unhappy couples can incorporate multiple negative emotions (e.g., anger, contempt, disgust or sadness) while sadness for instance can still indicate a happy couple which stands together in the face of external adversity. Reacting with appropriate affect in response to the partner may even signal motivation to resolve a conflict (Keltner \& Haidt, 2001) and also demonstrates responsiveness, as lack thereof has been repeatedly found to be associated with lasting effects of lower relationship functioning (see for a review Reis \& Gable, 2015). As shown in Fig. 3, DeepConnection was able to use additional body posture information, even if facial information was absent, which further differentiates our model from previous work. Lastly, our training data exhibited a large variation of background images which is more reminiscent of realworld scenarios and should pave the way for an application of DeepConnection.

Even with our limited computational resources, the inference time of DeepConnection per video frame was less than 0.15 seconds. This bodes well for near-real-time usage of DeepConnection in therapeutic and other settings, especially if provided with superior computational resources. Future work relying on a larger dataset of training images might benefit from exchanging the final dropout layer with the multi-sample dropout scheme proposed recently 


\section{CLASSIFYING RELATIONSHIP STATE FROM IMAGES OF COUPLES}

(Inoue, 2019). Achieving accelerated model convergence through averaging at this final layer, multi-sample dropout allowed us to train our DeepConnection model considerably faster without a loss in performance (Appendix Fig. 6). This is especially relevant, as the resource-intensive training of neural networks has recently been shown to negatively impact the environment (Strubell, Ganesh, \& McCallum, 2019), making efficient training routines paramount.

One clear limitation of the work presented here lies in the fact that most (though not all) of our training data consisted of stock images of couples and actors portraying couples in a specific emotional state. While this is advantageous for labeling and retrieving relevant images, one could argue that expressions seen in those images are exaggerated, artificial or unrelated to the behavior of real-world couples. If this holds true, it might detract from the applicability and generalizability of DeepConnection to real-world contexts. Here, it could be argued that actors frequently draw upon their own emotional experiences to portray said emotion, to the extent of actually feeling the respective emotion while acting (Kirby, 1972). This would lead to some degree of realistic representation and would result in better model performance on future tasks. Even an exaggeration of realistic gestures and facial expressions would allow the model to learn correctly and then assign real images with more moderately confident predictions.

Another limitation could be seen as a corollary to our reliance on a large fraction of stock images. Specifically, there is an underrepresentation of homosexual couples (around 2-4\% of the American population is homosexual, based on the National Survey of Sexual Health and Behavior, Chandra, Mosher, Copen, \& Sionean, 2011), of intercultural couples (28\%, U.S. Census Bureau, 2012), and of couples of color (e.g. 12.7\% of the population are African American, U.S. Census Bureau, 2016). Recent efforts in AI ethics have uncovered shortcomings and biases of effective deep learning models when it comes to recognizing people of color or 


\section{CLASSIFYING RELATIONSHIP STATE FROM IMAGES OF COUPLES}

women because their training data did not properly reflect the real world (Chen, Johansson, \& Sontag, 2018). Even ImageNet, the large-scale image dataset used to pretrain ResNet-34 used here has been shown to be biased toward images from the Northern Hemisphere (Tommasi, Patricia, Caputo, \& Tuytelaars, 2015). The majority of the images used for training here are of attractive, Caucasian, heterosexual couples of young to middle age. We realize that this is not necessarily representative of most contexts. Our dataset comprised limited amounts of images of homosexual (2.2\%) and non-Caucasian couples (15.64\%) and therefore only partially reflects the prevalence of homosexual and interracial couples in the US. Yet research has demonstrated that heterosexual and homosexual couples tend be similar regarding relationship quality (Kurdek, 2005; Peplau \& Fingerhut, 2007).

There are cultural differences regarding the appropriateness of emotion expression and which emotions individuals tend to respond to (Grossmann, Ellsworth, \& Hong, 2012). In more collectivistic countries, expressing anger is considered impolite and perceived as threatening to group harmony (Kitayama, Mesquita, \& Karasawa, 2006). Although norms about displaying emotions differ between Western and collectivistic cultures, emotional experience itself seems to be similar across cultures, as indicated by a study about physiological arousal (Tsai, Knutson, \& Fung, 2006). Gestures also vary by culture, and the same gesture can express friendliness in one country and an insult in another (Black, 2011). Beside these differences, basic emotions (anger, fear, sadness, surprise, disgust, and happiness) are considered universal and are displayed crossculturally in the same manner (Ekman \& Friesen, 1975). How couples express and experience emotions during interactions, or cope with conflicts, is likely to affect the relationship state of a couple, ultimately determining the stability of their relationship (Karney \& Bradbury, 1995). 


\section{CLASSIFYING RELATIONSHIP STATE FROM IMAGES OF COUPLES}

Potential ways forward would be the utilization of the massive amounts of unlabeled images already present on social media and other platforms. Recent advances in unsupervised and self-supervised deep learning could make this possible, such as the recent impressive demonstration of contrastive predictive coding (CPC) which allowed the usage of massive amounts of unlabeled images for classification purposes (Hénaff, Razavi, Doersch, Eslami, \& Oord, 2019). With couples uploading orders of magnitudes more images than we used in this study, deeper and more powerful neural networks could be trained which might lead to improved performance. One of the reasons why we decided to use ResNet-34 instead of, for instance, ResNet-101 as the base of DeepConnection was that we did not see a reliable increase in performance, possibly because of limited amounts of data. Additionally, this might offer a less artificial data source than web-scraped stock photos and increase generalizability (even though we realize that social media photos oftentimes are nearly as carefully staged as respective stock images and are also biased in terms of which groups of people upload these pictures). One study, for instance, found that individuals in relationships posting dyadic profile pictures on Facebook were more satisfied in their relationship than individuals posting pictures without their partner (Saslow, Muise, Impett, \& Dubin, 2013). One cautionary point here would be that the overwhelming majority of uploaded images will likely be of happy couples, as most people are unwilling to publicly share relationship conflicts or dissatisfaction with one's partner and commonly do not document such events with pictures.

\section{Code availability}

Trained models and all relevant code can be found at https://github.com/Bribak/DeepConnection. 


\section{CLASSIFYING RELATIONSHIP STATE FROM IMAGES OF COUPLES}

\section{References}

Ainsworth, M. D. S., \& Bell, S. M. (1970). Attachment, Exploration, and Separation: Illustrated by the Behavior of One-Year-Olds in a Strange Situation. Child Development, 41(1), 49. https://doi.org/10.2307/1127388

Barrett, L. F., Robin, L., Pietromonaco, P. R., \& Eyssell, K. M. (1998). Are Women the "More Emotional" Sex? Evidence From Emotional Experiences in Social Context. Cognition \& Emotion, 12(4), 555-578. https://doi.org/10.1080/026999398379565

Black, R. M. (2011). Cultural Considerations of Hand Use. Journal of Hand Therapy, 24(2), 104-111. https://doi.org/10.1016/j.jht.2010.09.067

Bowlby, J. (1988). A secure base: Parent-child attachment and healthy human development. New York, NY, US: Basic Books.

Butler, E. A. (2011). Temporal Interpersonal Emotion Systems: The "TIES" That Form Relationships. Personality and Social Psychology Review, 15(4), 367-393. https://doi.org/10.1177/1088868311411164

Carrere, S., \& Gottman, J. M. (1999). Predicting Divorce among Newlyweds from the First Three Minutes of a Marital Conflict Discussion. Family Process, 38(3), 293-301. https://doi.org/10.1111/j.1545-5300.1999.00293.x

Carstensen, L. L., Gottman, J. M., \& Levenson, R. W. (1995). Emotional behavior in long-term marriage. Psychology and Aging, 10(1), 140-149. https://doi.org/10.1037/0882-7974.10.1.140

Chandra, A., Mosher, W. D., Copen, C., \& Sionean, C. (2011). Sexual behavior, sexual attraction, and sexual identity in the United States: Data from the 2006-2008 National Survey of Family Growth. National Health Statistics Reports, (36), 1-36.

Chen, I., Johansson, F. D., \& Sontag, D. (2018). Why Is My Classifier Discriminatory? ArXiv:1805.12002 [Cs, Stat]. Retrieved from http://arxiv.org/abs/1805.12002 


\section{CLASSIFYING RELATIONSHIP STATE FROM IMAGES OF COUPLES}

Coan, J. A., \& Gottman, J. M. (2007). The specific affect coding system (SPAFF). In Handbook of emotion elicitation and assessment (pp. 267-285).

Cohan, C. L., \& Bradbury, T. N. (1997). Negative life events, marital interaction, and the longitudinal course of newlywed marriage. Journal of Personality and Social Psychology, 73(1), 114-128. https://doi.org/10.1037/0022-3514.73.1.114

Dachapally, P. R. (2017). Facial Emotion Detection Using Convolutional Neural Networks and Representational Autoencoder Units. ArXiv:1706.01509 [Cs, Stat]. Retrieved from http://arxiv.org/abs/1706.01509

Deng, J., Dong, W., Socher, R., Li, L., Li, K., \& Fei-fei, L. (2009). Imagenet: A large-scale hierarchical image database. In CVPR.

Ekman, P., \& Friesen, W. V. (1975). Unmasking the face: A guide to recognizing emotions from facial clues. Oxford, England: Prentice-Hall.

Ekman, P., \& Friesen, W. V. (1978). Facial action coding system: Manual. Palo Alto, Calif.: Consulting Psychologists Press.

Fischer, A. H., \& Manstead, A. S. R. (2016). Social functions of emotion and emotion regulation. In Handbook of emotion (pp. 456-469).

Floyd, F. J., Baucom, D. H., Godfrey, J. J., \& Palmer, C. (1998). Observational Methods. In Comprehensive Clinical Psychology (pp. 1-21). https://doi.org/10.1016/B0080-4270(73)00223-6

Funk, J. L., \& Rogge, R. D. (2007). Testing the ruler with item response theory: Increasing precision of measurement for relationship satisfaction with the Couples Satisfaction Index. Journal of Family Psychology, 21(4), 572-583. https://doi.org/10.1037/0893-3200.21.4.572

Gable, S. L., Reis, H. T., Impett, E. A., \& Asher, E. R. (2004). What Do You Do When Things Go Right? The Intrapersonal and Interpersonal Benefits of Sharing Positive Events. Journal of Personality and Social Psychology, 87(2), 228-245. https://doi.org/10.1037/0022-3514.87.2.228 


\section{CLASSIFYING RELATIONSHIP STATE FROM IMAGES OF COUPLES}

Glorot, X., \& Bengio, Y. (2010). Understanding the difficulty of training deep feedforward neural networks. Proceedings of the Thirteenth International Conference on Artificial Intelligence and Statistics, 249-256. Retrieved from http://proceedings.mlr.press/v9/glorot10a.html

Gottman, John M., Coan, J., Carrere, S., \& Swanson, C. (1998). Predicting marital happiness and stability from newlywed interactions. Journal of Marriage and the Family, 60(1), 5-22. https://doi.org/10.2307/353438

Gottman, John M., Murray, J. D., Swanson, C. C., Tyson, R., \& Swanson, K. R. (2002). The mathematics of marriage: Dynamic nonlinear models. Cambridge, MA, US: MIT Press.

Gottman, John Mordechai, Levenson, R. W., Gross, J., Frederickson, B. L., McCoy, K., Rosenthal, L., ... Yoshimoto, D. (2003). Correlates of Gay and Lesbian Couples’ Relationship Satisfaction and Relationship Dissolution. Journal of Homosexuality, 45(1), $23-43$. https://doi.org/10.1300/J082v45n01_02

Grandey, A., Rafaeli, A., Ravid, S., Wirtz, J., \& Steiner, D. D. (2010). Emotion display rules at work in the global service economy: The special case of the customer. Journal of Service Management, 21(3), 388-412. https://doi.org/10.1108/09564231011050805

Gross, J. J., \& John, O. P. (2003). Individual differences in two emotion regulation processes: Implications for affect, relationships, and well-being. Journal of Personality and Social Psychology, 85(2), 348-362. https://doi.org/10.1037/0022-3514.85.2.348

Grossmann, I., Ellsworth, P. C., \& Hong, Y. (2012). Culture, attention, and emotion. Journal of Experimental Psychology: General, 141(1), 31-36. https://doi.org/10.1037/a0023817

Gu, Y., Mai, X., \& Luo, Y. (2013). Do Bodily Expressions Compete with Facial Expressions? Time Course of Integration of Emotional Signals from the Face and the Body. PLoS ONE, 8(7), e66762. https://doi.org/10.1371/journal.pone.0066762 


\section{CLASSIFYING RELATIONSHIP STATE FROM IMAGES OF COUPLES}

He, K., Zhang, X., Ren, S., \& Sun, J. (2014). Spatial Pyramid Pooling in Deep Convolutional Networks for Visual Recognition. ArXiv:1406.4729 [Cs], 8691, 346-361. https://doi.org/10.1007/978-3319-10578-9_23

He, K., Zhang, X., Ren, S., \& Sun, J. (2015). Deep Residual Learning for Image Recognition. ArXiv:1512.03385 [Cs]. Retrieved from http://arxiv.org/abs/1512.03385

Hénaff, O. J., Razavi, A., Doersch, C., Eslami, S. M. A., \& Oord, A. van den. (2019). Data-Efficient Image Recognition with Contrastive Predictive Coding. ArXiv:1905.09272 [Cs]. Retrieved from http://arxiv.org/abs/1905.09272

Houben, M., Van Den Noortgate, W., \& Kuppens, P. (2015). The relation between short-term emotion dynamics and psychological well-being: A meta-analysis. Psychological Bulletin, 141(4), 901930. https://doi.org/10.1037/a0038822

Huang, G., Liu, Z., van der Maaten, L., \& Weinberger, K. Q. (2016). Densely Connected Convolutional Networks. ArXiv:1608.06993 [Cs]. Retrieved from http://arxiv.org/abs/1608.06993

Inoue, H. (2019). Multi-Sample Dropout for Accelerated Training and Better Generalization. ArXiv:1905.09788 [Cs, Stat]. Retrieved from http://arxiv.org/abs/1905.09788

Ioffe, S., \& Szegedy, C. (2015). Batch Normalization: Accelerating Deep Network Training by Reducing Internal Covariate Shift. ArXiv:1502.03167 [Cs]. Retrieved from http://arxiv.org/abs/1502.03167

Johnson, S. M., Hunsley, J., Greenberg, L., \& Schindler, D. (2006). Emotionally Focused Couples Therapy: Status and Challenges. Clinical Psychology: Science and Practice, 6(1), 67-79. https://doi.org/10.1093/clipsy.6.1.67

Karney, B. R., \& Bradbury, T. N. (1995). The longitudinal course of marital quality and stability: A review of theory, methods, and research. Psychological Bulletin, 118(1), 3-34. https://doi.org/10.1037/0033-2909.118.1.3 


\section{CLASSIFYING RELATIONSHIP STATE FROM IMAGES OF COUPLES}

Kashdan, T. B., Volkmann, J. R., Breen, W. E., \& Han, S. (2007). Social anxiety and romantic relationships: The costs and benefits of negative emotion expression are context-dependent. Journal of Anxiety Disorders, 21(4), 475-492. https://doi.org/10.1016/j.janxdis.2006.08.007

Keltner, D., \& Haidt, J. (2001). Social functions of emotions. In Emotions and Social Behavior. Emotions: Currrent issues and future directions (pp. 192-213). New York, NY, US: Guilford Press.

Kingma, D. P., \& Ba, J. (2014). Adam: A Method for Stochastic Optimization. ArXiv:1412.6980 [Cs]. Retrieved from http://arxiv.org/abs/1412.6980

Kirby, M. (1972). On Acting and Not-Acting. The Drama Review: TDR, 16(1), 3. https://doi.org/10.2307/1144724

Kirschbaum, C., Pirke, K.-M., \& Hellhammer, D. H. (1993). The 'Trier Social Stress Test' - A Tool for Investigating Psychobiological Stress Responses in a Laboratory Setting. Neuropsychobiology, 28(1-2), 76-81. https://doi.org/10.1159/000119004

Kitayama, S., Mesquita, B., \& Karasawa, M. (2006). Cultural affordances and emotional experience: Socially engaging and disengaging emotions in Japan and the United States. Journal of Personality and Social Psychology, 91(5), 890-903. https://doi.org/10.1037/0022-3514.91.5.890

Kret, M. E., Pichon, S., Grèzes, J., \& de Gelder, B. (2011). Similarities and differences in perceiving threat from dynamic faces and bodies. An fMRI study. NeuroImage, 54(2), 1755-1762. https://doi.org/10.1016/j.neuroimage.2010.08.012

Kurdek, L. A. (2005). What Do We Know About Gay and Lesbian Couples? Current Directions in Psychological Science, 14(5), 251-254. https://doi.org/10.1111/j.0963-7214.2005.00375.x

Kurien, D. N. (2010). Body language: Silent communicator at the workplace. 4, 29-36.

Laurenceau, J.-P., Barrett, L. F., \& Rovine, M. J. (2005). The Interpersonal Process Model of Intimacy in Marriage: A Daily-Diary and Multilevel Modeling Approach. Journal of Family Psychology, 19(2), 314-323. https://doi.org/10.1037/0893-3200.19.2.314 


\section{CLASSIFYING RELATIONSHIP STATE FROM IMAGES OF COUPLES}

Laursen, B., \& Hafen, C. A. (2009). Future Directions in the Study of Close Relationships: Conflict Is Bad (Except When It's Not): Conflict Is Bad (Except When It's Not). Social Development, 19(4), 858-872. https://doi.org/10.1111/j.1467-9507.2009.00546.x

Lecun, Y., Bottou, L., Bengio, Y., \& Haffner, P. (1998). Gradient-based learning applied to document recognition. Proceedings of the IEEE, 86(11), 2278-2324. https://doi.org/10.1109/5.726791

Li, S., \& Deng, W. (2018). Deep Facial Expression Recognition: A Survey. ArXiv:1804.08348 [Cs]. Retrieved from http://arxiv.org/abs/1804.08348

Li, Z., Dekel, T., Cole, F., Tucker, R., Snavely, N., Liu, C., \& Freeman, W. T. (2019). Learning the Depths of Moving People by Watching Frozen People. ArXiv:1904.11111 [Cs]. Retrieved from http://arxiv.org/abs/1904.11111

Loshchilov, I., \& Hutter, F. (2016). SGDR: Stochastic Gradient Descent with Warm Restarts. ArXiv:1608.03983 [Cs, Math]. Retrieved from http://arxiv.org/abs/1608.03983

Luginbuehl, T., \& Schoebi, D. (2019). Emotion dynamics and responsiveness in intimate relationships. Emotion. https://doi.org/10.1037/emo0000540

Mikolajczyk, A., \& Grochowski, M. (2018). Data augmentation for improving deep learning in image classification problem. 2018 International Interdisciplinary PhD Workshop (IIPhDW), 117-122. https://doi.org/10.1109/IIPHDW.2018.8388338

Mohammadpour, M., Khaliliardali, H., Hashemi, S. Mohammad. R., \& AlyanNezhadi, Mohammad. M. (2017). Facial emotion recognition using deep convolutional networks. 2017 IEEE 4th International Conference on Knowledge-Based Engineering and Innovation (KBEI), 0017-0021. https://doi.org/10.1109/KBEI.2017.8324974

Nair, V., \& Hinton, G. (2010). Rectified Linear Units Improve Restricted Boltzmann Machines. Proceedings of the 27th International Conference on International Conference on Machine Learning, 807-814. 


\section{CLASSIFYING RELATIONSHIP STATE FROM IMAGES OF COUPLES}

Parkinson, B. (2001). Putting appraisal in context. In Series in affective science. Appraisal processes in emotion: Theory, methods, research (pp. 173-186).

Paszke, A., Gross, S., Chintala, S., Chanan, G., Yang, E., DeVito, Z., ... Lerer, A. (2017). Automatic Differentiation in PyTorch. In NIPS Autodiff Workshop.

Peplau, L. A., \& Fingerhut, A. W. (2007). The Close Relationships of Lesbians and Gay Men. Annual Review of Psychology, 58(1), 405-424. https://doi.org/10.1146/annurev.psych.58.110405.085701

Prechelt, L. (2012). Early Stopping — But When? In G. Montavon, G. B. Orr, \& K.-R. Müller (Eds.), Neural Networks: Tricks of the Trade (Vol. 7700, pp. 53-67). https://doi.org/10.1007/978-3-64235289-8_5

Reis, H. T., \& Gable, S. L. (2015). Responsiveness. Current Opinion in Psychology, 1, 67-71. https://doi.org/10.1016/j.copsyc.2015.01.001

Ronneberger, O., Fischer, P., \& Brox, T. (2015). U-Net: Convolutional Networks for Biomedical Image Segmentation. ArXiv:1505.04597 [Cs]. Retrieved from http://arxiv.org/abs/1505.04597

Roseman, I. J., Wiest, C., \& Swartz, T. S. (1994). Phenomenology, behaviors, and goals differentiate discrete emotions. Journal of Personality and Social Psychology, 67(2), 206-221. https://doi.org/10.1037/0022-3514.67.2.206

Saslow, L. R., Muise, A., Impett, E. A., \& Dubin, M. (2013). Can You See How Happy We Are? Facebook Images and Relationship Satisfaction. Social Psychological and Personality Science, 4(4), 411-418. https://doi.org/10.1177/1948550612460059

Selvaraju, R. R., Cogswell, M., Das, A., Vedantam, R., Parikh, D., \& Batra, D. (2016). Grad-CAM: Visual Explanations from Deep Networks via Gradient-based Localization. ArXiv:1610.02391 [Cs]. Retrieved from http://arxiv.org/abs/1610.02391

Shaver, P., Hazan, C., \& Bradshaw, D. (1988). Love as attachment. In The psychology of love (pp. 6899). New Haven, CT, US: Yale University Press. 


\section{CLASSIFYING RELATIONSHIP STATE FROM IMAGES OF COUPLES}

Shiota, M., Campos, B., Keltner, D., \& Hertenstein, M. J. (2004). Positive emotion and the regulation of interpersonal relationships. The Regulation of Emotion, 129-157. https://doi.org/10.4324/9781410610898

Srivastava, N., Hinton, G., Krizhevsky, A., Sutskever, I., \& Salakhutdinov, R. (2014). Dropout: A Simple Way to Prevent Neural Networks from Overfitting. Journal of Machine Learning Research, 15, $1929-1958$.

Strubell, E., Ganesh, A., \& McCallum, A. (2019). Energy and Policy Considerations for Deep Learning in NLP. ArXiv:1906.02243 [Cs]. Retrieved from http://arxiv.org/abs/1906.02243

Tommasi, T., Patricia, N., Caputo, B., \& Tuytelaars, T. (2015). A Deeper Look at Dataset Bias. ArXiv:1505.01257 [Cs]. Retrieved from http://arxiv.org/abs/1505.01257

Tsai, J. L., Knutson, B., \& Fung, H. H. (2006). Cultural variation in affect valuation. Journal of Personality and Social Psychology, 90(2), 288-307. https://doi.org/10.1037/0022-3514.90.2.288

Voulodimos, A., Doulamis, N., Doulamis, A., \& Protopapadakis, E. (2018). Deep Learning for Computer Vision: A Brief Review. Computational Intelligence and Neuroscience, 2018, 1-13. https://doi.org/10.1155/2018/7068349

Webb, E. J., Campbell, D. T., Schwartz, R. D., \& Sechrest, L. (1966). Unobtrusive measures: Nonreactive research in the social sciences. Oxford, England: Rand Mcnally.

Xu, X., Li, G., Xie, G., Ren, J., \& Xie, X. (2019). Weakly Supervised Deep Semantic Segmentation Using CNN and ELM with Semantic Candidate Regions. Complexity, 2019, 1-12. https://doi.org/10.1155/2019/9180391

Yosinski, J., Clune, J., Bengio, Y., \& Lipson, H. (2014). How transferable are features in deep neural networks? ArXiv:1411.1792 [Cs]. Retrieved from http://arxiv.org/abs/1411.1792

Zhang, C.-L., \& Wu, J. (2019). Improving CNN linear layers with power mean non-linearity. Pattern Recognition, 89, 12-21. https://doi.org/10.1016/j.patcog.2018.12.029 


\section{Appendix}

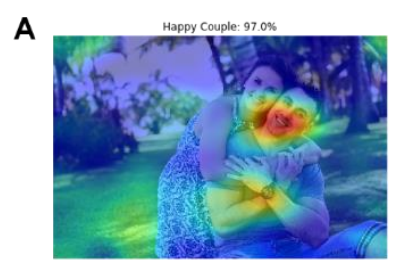

C

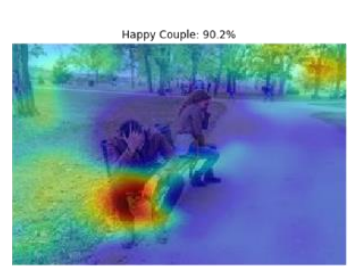

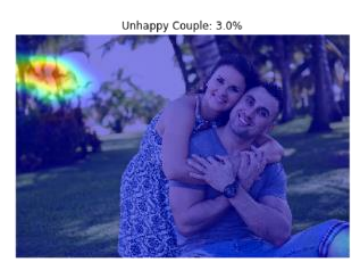

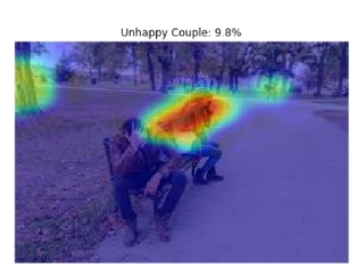

B

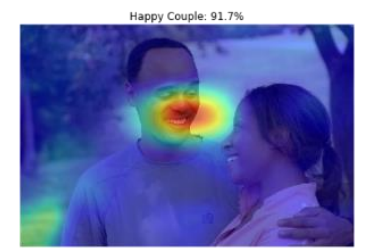

D

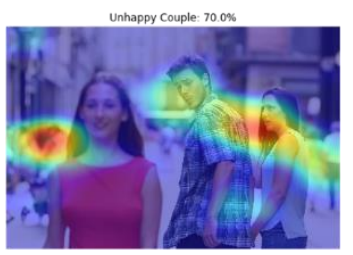

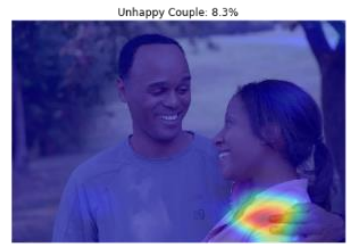

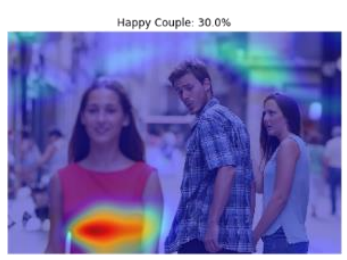

Figure 1. Interpretation of ResNet-34 model predictions using Grad-CAM. Four representative images of couples not used in training (two labeled as happy (A-B) and two as unhappy (C-D)) were used as inputs a trained ResNet-34 model. Gradients at the last convolutional layer were used to generate class-specific saliency heatmaps and layered on top of the original images together with the predicted class label and the model confidence in percent. Heatmaps of predicted class labels are always depicted as the left-hand image irrespective of the true label.

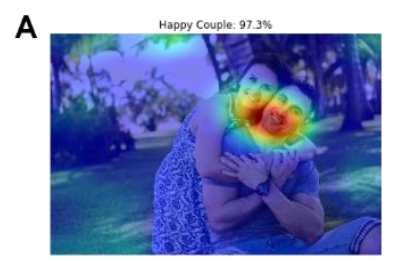

C

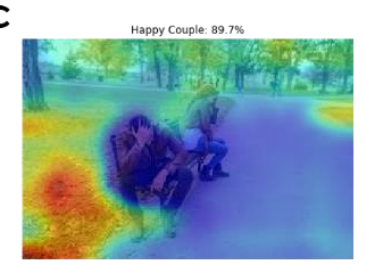

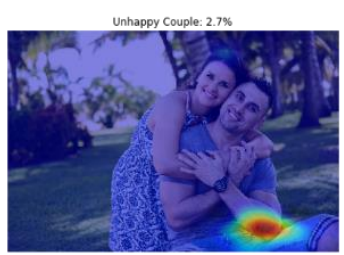

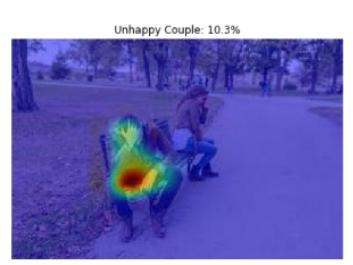

B

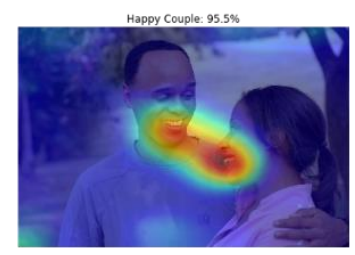

D

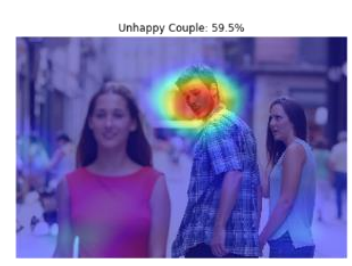

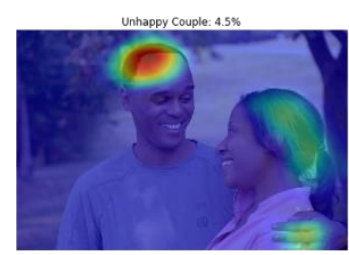

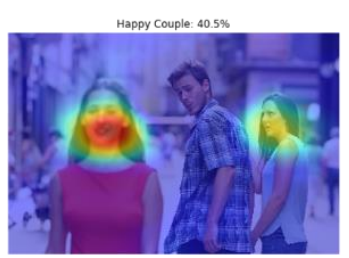




\section{CLASSIFYING RELATIONSHIP STATE FROM IMAGES OF COUPLES}

Figure 2. Interpretation of SPP model predictions using Grad-CAM. Four representative images of couples not used in training (two labeled as happy (A-B) and two as unhappy (C-D)) were used as inputs for a trained SPP model. Gradients at the last convolutional layer were used to generate class-specific saliency heatmaps and layered on top of the original images together with the predicted class label and the model confidence in percent. Heatmaps of predicted class labels are always depicted as the left-hand image irrespective of the true label.

A

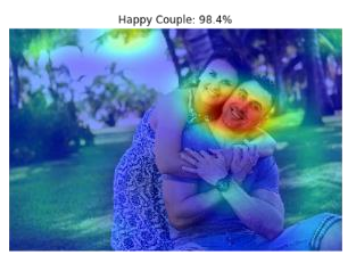

C

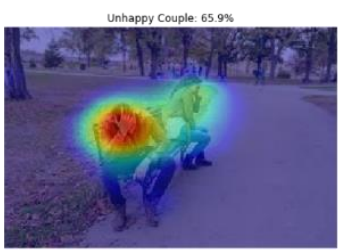

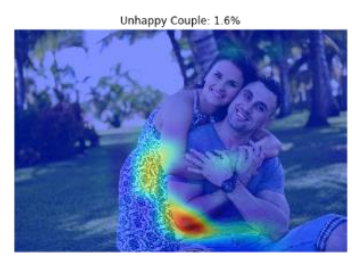

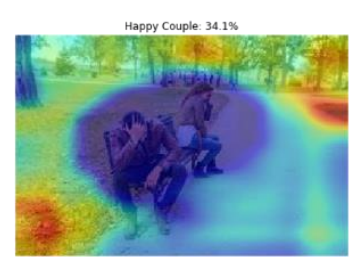

B

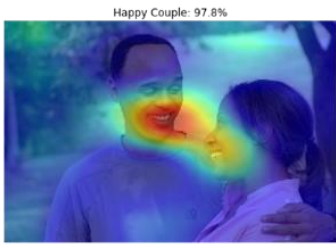

D

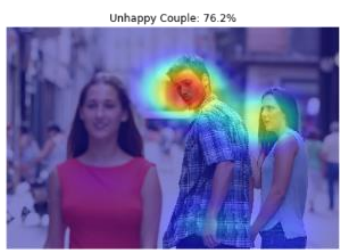

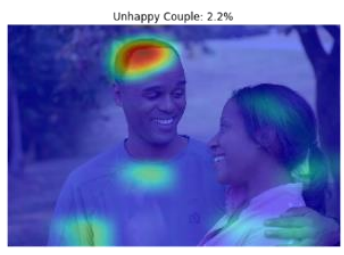

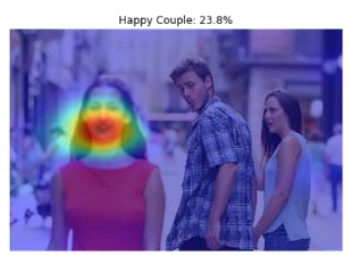

Figure 3. Interpretation of DeepConnection model predictions using Grad-CAM. Four representative images of couples not used in training (two labeled as happy (A-B) and two as unhappy (C-D)) were used as inputs for the trained DeepConnection model. Gradients at the last convolutional layer were used to generate class-specific saliency heatmaps and layered on top of the original images together with the predicted class label and the model confidence in percent. Heatmaps of predicted class labels are always depicted as the left-hand image irrespective of the true label. 

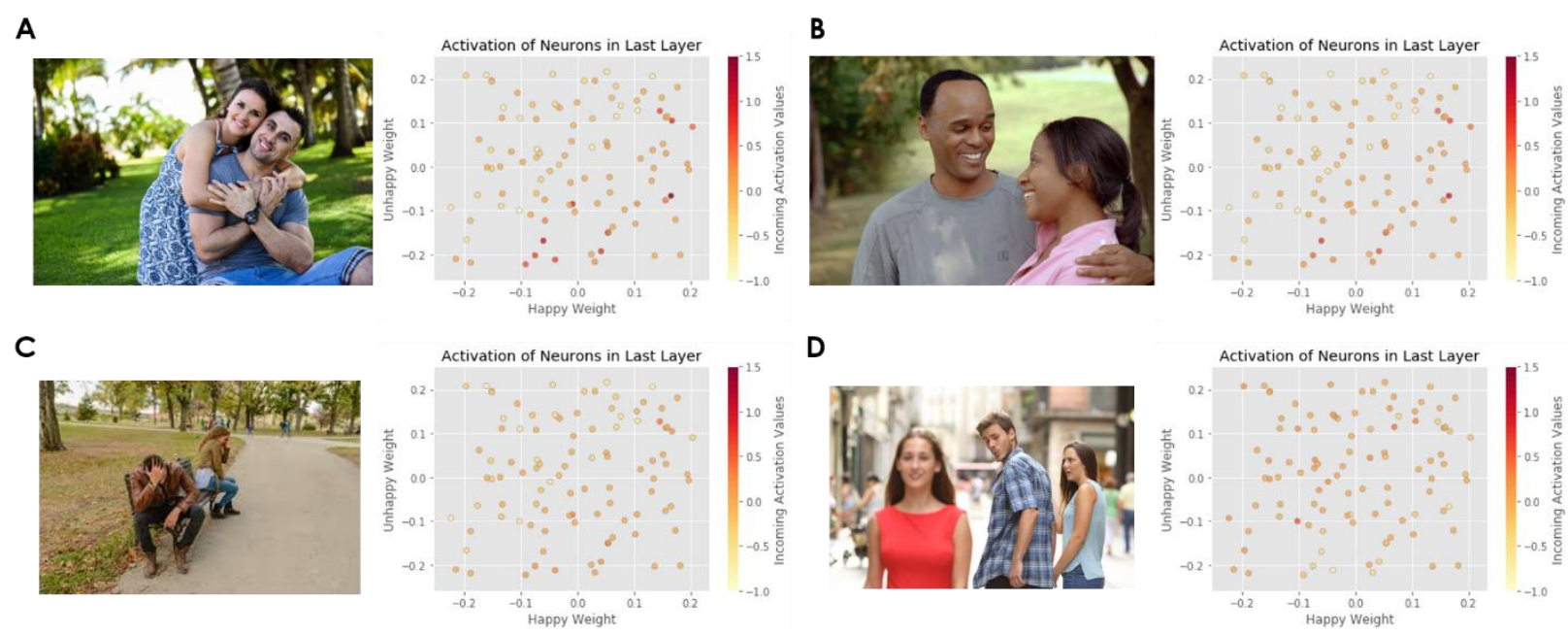

Figure 4. Activations and weights of the last fully connected layer of the SPP model for selected images. Four representative images of couples not used in training (two labeled as happy (A-B) and two as unhappy (C-D)) were used as inputs for the trained SPP model. Plotted points represent the weights of the last fully connected layer of size 100x2 in the trained SPP model which, together with their inputs, determine the class probabilities. Points were colored by the magnitude of activations from the presented image directly prior to the last fully connected layer. To determine class probabilities, activations will be multiplied by class weights and summed.

A

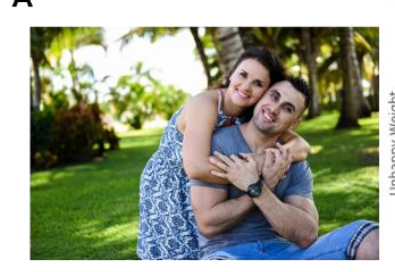

C

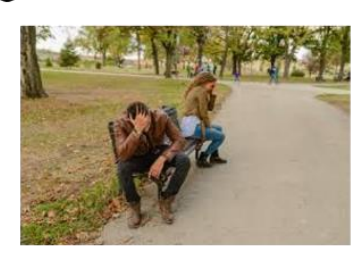

B
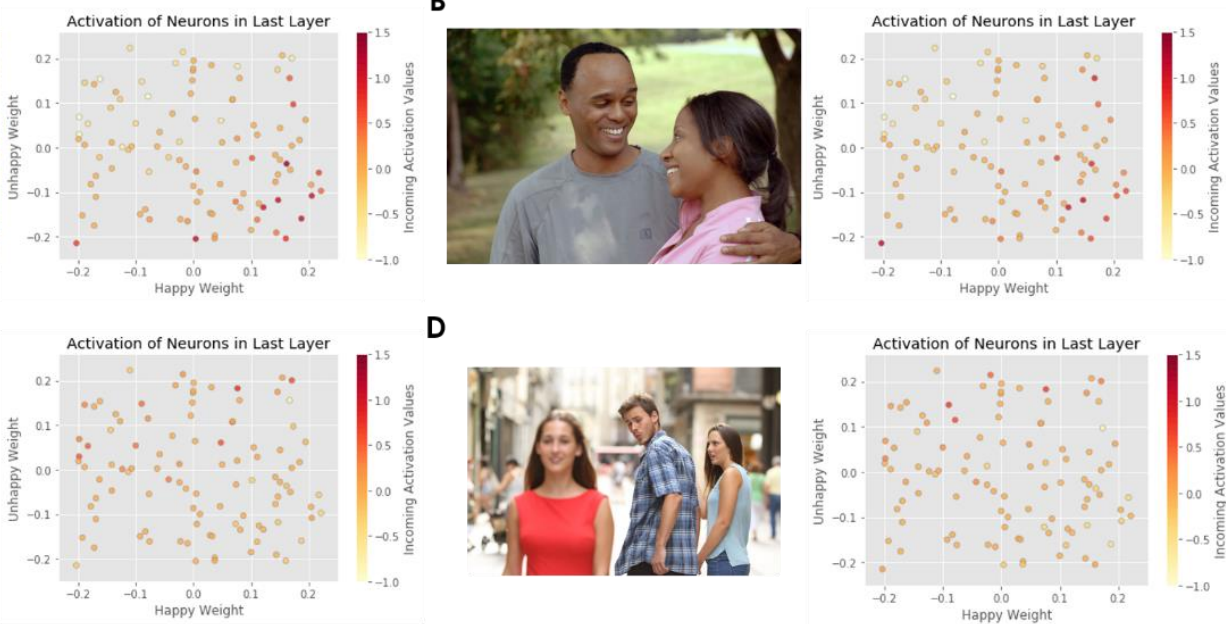

D

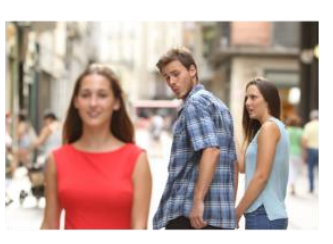

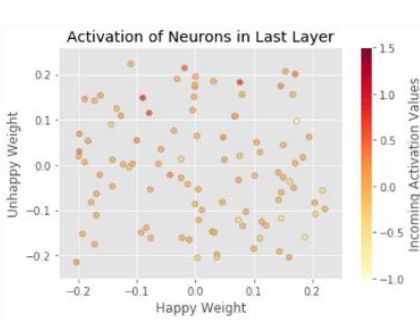




\section{CLASSIFYING RELATIONSHIP STATE FROM IMAGES OF COUPLES}

Figure 5. Activations and weights of the last fully connected layer of the DeepConnection model for selected images. Four representative images of couples not used in training (two labeled as happy (A-B) and two as unhappy (C-D)) were used as inputs for the trained DeepConnection model. Plotted points represent the weights of the last fully connected layer of size $100 \times 2$ in the trained DeepConnection model which, together with their inputs, determine the class probabilities. Points were colored by the magnitude of activations from the presented image directly prior to the last fully connected layer. To determine class probabilities, activations will be multiplied by class weights and summed.

A

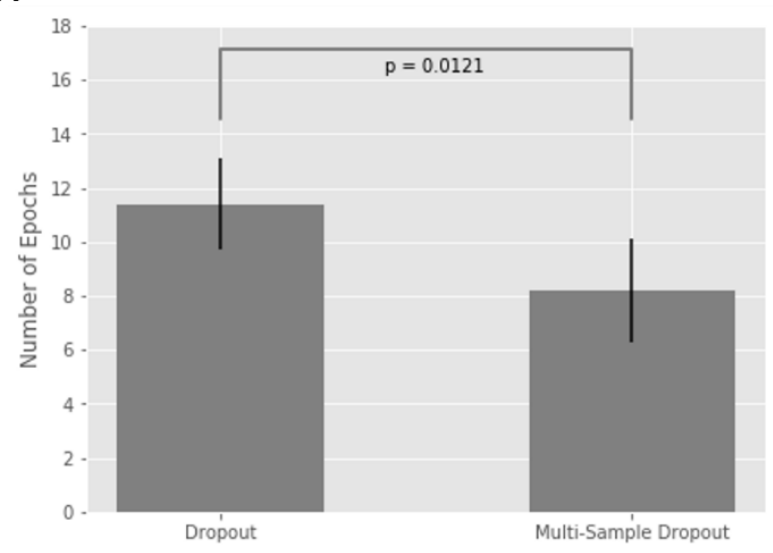

B

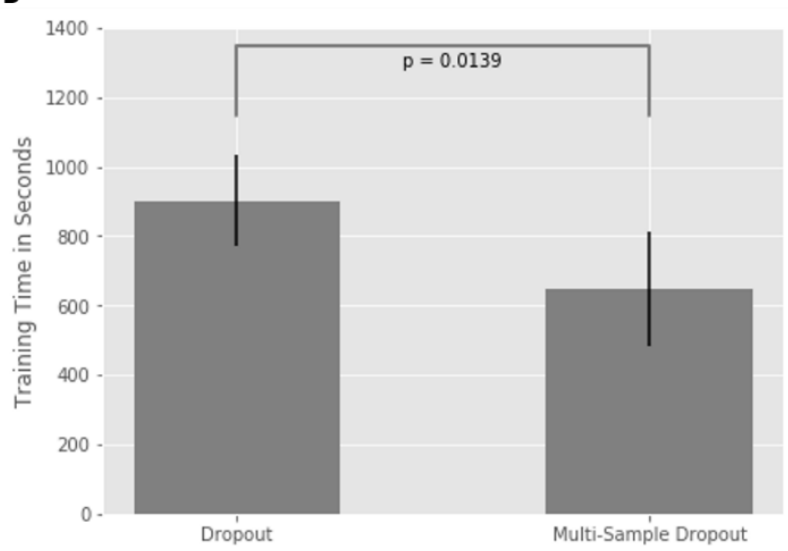

Figure 6. Training the DeepConnection model is accelerated by multi-sample dropout.

Exchanging the dropout layer immediately prior to the last fully connected layer in

DeepConnection with a multi-sample dropout layer (using eight dropout channels) enabled faster training convergence, both with regard to the number of epochs (A) as well as to the total amount of time required (B). Data shown was gathered from five training runs per dropout method and is plotted as mean \pm standard deviation. The calculation of $p$-values was done using a two-tailed Student's t-test. 